Louisiana State University

LSU Digital Commons

3-1-2016

\title{
Competition-mediated feedbacks in experimental multispecies epizootics
}

Tad Dallas

University of Georgia

Richard J. Hall

University of Georgia

John M. Drake

University of Georgia

Follow this and additional works at: https://digitalcommons.Isu.edu/biosci_pubs

\section{Recommended Citation}

Dallas, T., Hall, R., \& Drake, J. (2016). Competition-mediated feedbacks in experimental multispecies epizootics. Ecology, 97 (3), 661-670. https://doi.org/10.1890/15-0305

This Article is brought to you for free and open access by the Department of Biological Sciences at LSU Digital Commons. It has been accepted for inclusion in Faculty Publications by an authorized administrator of LSU Digital Commons. For more information, please contact ir@lsu.edu. 


\title{
Competition-mediated feedbacks in experimental multispecies epizootics
}

\author{
Tad Dallas, ${ }^{1,3}$ Richard J. Hall, ${ }^{1,2}$ and John M. Drake ${ }^{1}$ \\ ${ }^{1}$ Odum School of Ecology, University of Georgia, 140 E. Green Street, Athens, Georgia 30602, USA \\ ${ }^{2}$ Department of Infectious Disease, University of Georgia, 501 D. W. Brooks Drive, Athens, Georgia 30602, USA
}

\begin{abstract}
Competition structures ecological communities and alters host-pathogen interactions. In environmentally transmitted pathogens, an infection-resistant competitor may influence infection dynamics in a susceptible species through the negative impacts of competition (e.g., by reducing host density or causing nutritional stress that increases susceptibility to infection) and/or the positive impacts of reducing transmission efficiency (e.g., by removing environmental pathogen stages). Thus, a non-susceptible competitor may enhance, reduce, or have no net effect on susceptible host density and infection prevalence. Here, we couple an epidemiological model with experimental epidemics to test how resource competition with a non-susceptible competitor (Daphnia pulicaria) influences fungal microparasite (Metschnikowia bicuspidata) infection dynamics in a susceptible host species $(D$. dentifera). Our model and experiments suggest that competitor density can mediate the direction and magnitude of the effect of competition on infection dynamics, with a peak in infection prevalence occurring at intermediate competitor densities. At low densities, the non-susceptible competitor $D$. pulicaria may reduce infection prevalence in the susceptible host by removing fungal spores from the environment through feeding. However, when competitor density is increased and resources become limiting, D. pulicaria negatively impacts the susceptible host by increasing susceptible host feeding rates, and therefore fungal spore intake, and further by reducing susceptible host population size as it is driven toward competitive exclusion. In conclusion, these results show that a tradeoff between the competitor as a consumer of pathogen, which serves to reduce epidemic size, and as a modifier of susceptible host foraging ecology, which influences infection rates, may alternately enhance or dampen the magnitude of local epidemics.
\end{abstract}

Key words: competition; dilution effect; diversity-disease; environmentally transmitted pathogen; multihost pathogen; resource limitation.

\section{INTRODUCTION}

Competition is a major structuring force of ecological communities, and the strength of interactions between competitors can determine whether coexistence or competitive exclusion occurs (Armstrong and McGehee 1976; Wang et al. 2009). Further, environmental conditions can influence the outcome of competition, as competitive outcomes can be shaped by predators (Wollrab, de Roos and Diehl 2013), temperature (Carmona-Catot, Magellan and García-Berthou 2013), resource availability (Riebesell 1974), or natural enemies (Decaestecker et al. 2015). Studies of competition typically focus on the long-term or equilibrium dynamics of competing species (Wedin and Tilman 1993), but many interesting competitive interactions occur when populations are not at equilibrium, or are in the transient period before competitive coexistence or exclusion (Pickett 1980). Epidemic pathogens or parasites are a prime example of this, as

Manuscript received 19 February 2015; revised 3 September 2015; accepted 14 September 2015. Corresponding Editor: T. E. $\mathrm{X}$. Miller.

3E-mail: tdallas@uga.edu they disrupt host population dynamics, and can influence transient population dynamics and competitive outcomes. Pathogens may mediate the interactions between competing species by disproportionately affecting one of the competitors (Price, Westoby and Rice 1988), or by altering aspects of host life history such as development time or dispersal (Thomas et al. 2000). While the influence of parasites on competitive interactions has received ample attention (Park 1948; Price, Westoby and Rice 1988; Preston and Johnson 2010; Hatcher, Dick and Dunn 2012), there have been few studies into the role of competitors on parasite populations (Hall et al. 2009).

Competitors may differ in competence (Kilpatrick et al. 2006) and susceptibility to pathogen infection (Hall et al. 2007), such that the addition of a competitor can reduce, enhance, or have no net effect on infection dynamics in another competing host. For instance, a competitor that is a more competent host species could increase parasite population size, which would potentially elevate infection rates of the other competing host. However, if the competitor is a less competent host, or if the effect of competition reduces potential pathogen 
transmission events to the other competitor, infection risk may be reduced. The effect of competition therefore may offer a mechanistic explanation for some cases of the relationship between host diversity and disease risk (the "diversity-disease relationship").

The diversity-disease relationship proposes that increases in host diversity may reduce (i.e., a dilution effect) or enhance (i.e., an amplification effect) infection risk in a focal host species (Orlofske et al. 2012; Ostfeld and Keesing 2012). In theory, dilution effects may arise for many different reasons, but generally, the non-focal species are considered to be subject to "wasted" transmission events, so that pathogen fitness is reduced by infecting a less suitable host (Keesing, Holt and Ostfeld 2006). Studies of the dilution effect are typically phenomenological (Salkeld, Padgett and Jones 2013), and do not incorporate ecological interactions among species in the community. The inclusion of ecological interactions (e.g., competition) into studies of multi-host pathogen dynamics may inform a general theory for when we expect host diversity to reduce or enhance disease. Here, we use a combination of modeling and experiments to provide a link between diversity-disease relationships and parasite-mediated competition. Specifically, we investigate the impact of the addition of a non-susceptible superior competitor that consumes environmental pathogen on the infection and population dynamics of a susceptible host species.

To do this, we use a model system comprised of two sympatric zooplankton competitors, Daphnia dentifera and Daphnia pulicaria. These two species have been found to co-occur in the north temperate lakes of the United States (Duffy et al. 2010). Daphnia dentifera is susceptible to infection by an environmentally transmitted yeast pathogen (Metschnikowia bicuspidata), and is also an inferior competitor to D. pulicaria, as D. pulicaria has larger body size, reproductive rate (unpublished data), and foraging rate (Gliwicz 2004). Further, D. pulicaria has been found to outcompete D. lumholtzi, a formidable invasive competitor (Engel and Tollrian 2011). While D. pulicaria does not become infected, it does consume pathogen spores during foraging, potentially reducing pathogen transmission to susceptible hosts (so-called "friendly competition"; Hall et al. 2009). Reduced resources can nutritionally stress susceptible hosts, which can result in enhanced pathogen transmission (Pulkkinen and Ebert 2004) as a result of increased filtering rate (Hall et al. 2007), providing a mechanistic link between host foraging ecology and pathogen transmission. Therefore, the impact of competition on infection dynamics will depend on the tradeoff between the role of the competitor as a consumer-of-pathogen and as a consumer-of-resources.

Previously, Hall et al. (2009) performed an experiment in which $D$. pulicaria were allowed to graze on pathogen spores, and then this media was exposed to susceptible $D$. dentifera to see if $D$. pulicaria grazing could reduce transmission by depleting environmental pathogen spores.
However, this study did not account for the role of the competitor (D. pulicaria) as a consumer. By reducing algal resources, the competitor may indirectly influence susceptible host foraging rate, which is intrinsically linked to pathogen transmission in this system. A theory for this complex of interactions was recently developed by Cáceres et al. (2014), who examined the equilibrium outcomes of competition between a susceptible and a non-susceptible competitor, finding competitive exclusion of the susceptible host species over long time scales when the non-susceptible species is a superior competitor. While previous studies of parasite-mediated competition have focused on directly transmitted pathogens, and superior competitors that are also susceptible to parasitism (Price et al. 1986; Price, Westoby and Rice 1988), we focus on an environmentally transmitted pathogen, and the interaction between a dominant competitor that does not become infected and an inferior susceptible competitor. Theory predicts competitive exclusion of the inferior competitor in the long-term, although these species coexist in natural systems, most likely through niche partitioning or complex community interactions. We focus on the transient period where both species coexist, and examine infection dynamics as a result of competition for a limiting resource altering exposure to an environmentally transmitted pathogen. These transient dynamics are important, and ecologically relevant, given that the seasonal fluctuations in both zooplankton population sizes and infection dynamics may preclude zooplankton populations from achieving equilibrium dynamics (Hutchinson 1961; Scheffer et al. 2003).

Using a modified version of Cáceres et al. (2014) model that more closely matches our experimental system (see Appendix S1 for a comparison of our model to Cáceres et al. [2014]), we extend this theory by examining the influence of competitor density on epidemic and population dynamics under non-equilibrium conditions. By examining the transient dynamics of our theoretical model, we generate several testable model predictions, and experimentally test these predictions using the same zooplankton-pathogen system examined by Hall et al. (2007). First, we predict that the extent to which the competitor enhances or reduces infection prevalence in the susceptible host will depend on the initial density of the competitor and the availability of algal resources. When resources are limiting, we predict that the competitor will enhance infection prevalence in the susceptible host species by enhancing susceptible host foraging rate, and subsequent pathogen transmission. Second, we predict that susceptible host population size will decrease as a function of competitor density, since the susceptible host will be excluded more rapidly at higher densities of the superior competitor. This reduction in susceptible host population size may reduce infection prevalence if contact with pathogen (and therefore transmission) is reduced, or increase infection prevalence if susceptible host filtering rate is increased as a response to reduced 
resources. To test these hypotheses, we parameterized our epidemiological model, and compared model outputs with experimental epidemics. Experimental epidemics were initiated at three competitor densities, as increased competitor density serves to reduce resource availability through exploitative competition. We found that competition rarely benefited the susceptible host species, either enhancing infection prevalence at intermediate competitor densities, or competitively excluding the susceptible host at high competitor densities. This work highlights the importance of competitive interactions in evaluating the direction of diversity-disease relationships.

\section{Methods}

\section{Study system}

Clonal lines of two sympatric freshwater cladocerans were used in this study, D. dentifera (provided by M. Duffy) and D. pulicaria (originally isolated from Oneida Lake, New York, and provided by N. Hairston, Jr.). Metschnikowia bicuspidata is a fungal pathogen that infects $D$. dentifera, but not $D$. pulicaria. Pathogen transmission can occur when the host ingests the pathogen, allowing the pathogen to pierce the gut wall and grow within the host. Parasite-induced mortality causes the release of a multitude of infectious spores (see Table 1), which are then filtered and ingested by other hosts. Recent studies have found essentially no genetic variation in the fungal pathogen, reducing the likelihood of genotype $\times$ genotype interactions (Searle et al. 2015). However, genetic variation within natural $D$. dentifera populations could influence pathogen transmission dynamics through heterogeneity in resistance, or spore production per host (Carius et al. 2001; Auld et al. 2013). We acknowledge this as an interesting avenue for further research. However, our focus is to elucidate patterns in infection dynamics due to competitor density. Therefore, we selected a single, well-studied $D$. dentifera clone with moderate susceptibility to infection (Dallas and Drake 2014) for our experiments. Model sensitivity analyses (Appendix S1) further suggest that our qualitative results are robust to variation in plausible ranges of host infection parameters.

\section{Epidemiological model}

To examine the impact of a competitor on susceptible host infection dynamics, we used a two-host compartmental model, where the susceptible host species may be uninfected $(S)$ or infected $(I)$ by an environmentally transmitted fungal pathogen (with free-living spore population size $P$ ). This susceptible host species competes for resources $(R)$ with a non-susceptible competitor $(C)$. The model was formulated to correspond directly to the experimental treatments, allowing for the testing of model predictions with experimental data. The demographic and epidemic dynamics are described by the following system of differential equations:

$$
\begin{gathered}
\dot{S}=e_{S} f_{S}(R) R(S+I \phi)-\mu_{S} S-u f_{S}(R) S P \\
\dot{I}=u f_{S}(R) S P-\mu_{I} I \\
\dot{P}=\theta \mu_{I} I-\mu_{P} P-z_{S} f_{S}(R)(S+I) P-z_{C} f_{C}(R) C P \\
\dot{R}=\pi-\mu_{R} R-f_{S}(R)(S+I) R-f_{C}(R) C R
\end{gathered}
$$

\begin{tabular}{|c|c|c|c|c|}
\hline Variable & Units & Definition & Value & Citation \\
\hline$e_{\mathrm{S}}$ & unitless & assimilation efficiency (Susceptible) & 26 & \\
\hline$e_{\mathrm{C}}$ & unitless & assimilation efficiency (Competitor) & 28 & \\
\hline$\mu_{\mathrm{S}}$ & $\mathrm{d}^{-1}$ & death rate (Susceptible) & 0.10 & 1 \\
\hline $\mathrm{u}_{\mathrm{I}}$ & $d^{-1}$ & death rate (Infected) & 0.15 & 2 \\
\hline$\mu_{\mathrm{C}}$ & $d^{-1}$ & death rate (Competitor) & 0.10 & \\
\hline$\mu_{\mathrm{P}}$ & $d^{-1}$ & death rate (Pathogen) & 0.25 & 3 \\
\hline$\mu_{\mathrm{R}}$ & $\mathrm{d}^{-1}$ & death rate (Resource) & 0.25 & \\
\hline$z_{\mathrm{S}}$ & unitless & fraction spores digested (Susceptible) & 0.30 & \\
\hline${ }_{\mathrm{C}}$ & unitless & fraction spores digested (Competitor) & 0.30 & \\
\hline$\varphi$ & unitless & fecundity reduction by infection & 0.75 & 2 \\
\hline$f_{\mathrm{S} 0}, f_{\mathrm{S} 1}$ & $\mathrm{ml} \mathrm{d}^{-1}$ & host filtering rate (Suscep tible) & $0.020,4$ & 4,5 \\
\hline$f_{\mathrm{C} 0}, f_{\mathrm{C} 1}$ & $\mathrm{ml} \mathrm{d}^{-1}$ & host filtering rate (Competitor) & $0.025,4$ & 4,5 \\
\hline$u$ & unitless & per spore infectivity & $2.03 \times 10^{-4}$ & 4 \\
\hline$\theta$ & no. spores & mean spore load per infected host & $2 \times 10^{4}$ & 3 \\
\hline$\pi$ & $m g \mathrm{~L}^{-1} \mathrm{~d}^{-1}$ & resource supply rate & $0.005-4$ & 6 \\
\hline
\end{tabular}

TABle 1. Parameters, definitions, and units used in our epidemiological model

1: Stich and Maier (2007); 2: Duffy and Hall (2008); 3: Dallas and Drake (2014); 4: Hall et al. (2010); 5: DeMott (1982); 6 : Tessier and Woodruff (2002). We chose plausible estimates for parameters for which data were not readily available (denoted by an empty citation column). Our values of assimilation efficacy were estimated using information on Daphnia population growth rates (Smith 1963; Civitello et al. 2013). 


$$
\dot{C}=e_{C} f_{C}(R) R C-\mu_{C} C .
$$

Susceptible $(S)$ and competitor $(C)$ populations grow proportionally to the rate at which individuals can acquire $\left(f_{j}(R), j=S, C\right)$ and assimilate $\left(e_{j}, j=S, C\right)$ resources, die at rate $\mu_{S}$ (susceptible) or $\mu_{C}$ (competitor), and become infected at a rate determined by their filtering rate $\left(f_{j}(R), j=S, C\right)$ and a per spore infectivity parameter $(u)$. Filtering rates depend on the density of algal resources $(R)$ (Hall et al. 2010; Cáceres et al. 2014) and filtering alters the rate of environmental pathogen and resource loss from the system, as well as the transmission of pathogen. Infected $(I)$ individuals still produce susceptible offspring, but at a rate reduced by $\varphi$. Infected individuals die at rate $\mu_{I}>\mu_{S}$ to account for pathogen-induced mortality (i.e., virulence). Upon death, hosts release a burst of pathogen spores $(\theta)$ to the environmental pathogen bank. Environmental pathogen $(P)$ decays at a constant rate $\mu_{P}$, and is also ingested by susceptible $(S)$, infected $(I)$, and competitor $(C)$ individuals at rates determined by their corresponding filtering rates $\left(f_{j}(R), j=S, C\right)$, and a parameter which determines the fraction of spores ingested that are rendered non-infectious after passage through the host gut $\left(z_{j}, j=S, C\right)$; this matches observations of spore survival after bluegill feeding (Duffy 2009), and Daphnia hosts exposed to Pasteuria ramosa, a bacterial parasite (King et al. 2013). Resource $(R)$ is introduced at a constant rate $(\pi)$, and decays at a per capita rate $\mu_{R}$ plus additional decay as a function of host foraging $\left(f_{j}(R), j=S, C\right)$.

While the exact relationship between algal resource concentration and Daphnia filtering rate is unclear, evidence suggests that clearance rate is negatively related to algal resource quantity, such that it is highest when algal resources are limiting (Porter, Gerritsen and Orcutt 1982; Hall et al. 2007; Sarnelle and Wilson 2008; Hall et al. 2010). Therefore, we use a type II functional response for filtering rates (Eq. 6 and 7). To establish the competitor as dominant, we increased the competitor's assimilation coefficient $(e)$ and maximum filtering rate $\left(f_{C 0}\right)$ relative to the susceptible host species, reflecting the biology of the system, as the competitor is a larger-bodied grazer with an elevated filtering rate, larger clutch sizes, and faster growth. The equations for host species and competitor filtering rates are provided below, where $f_{S O}$ and $f_{C O}$ are the maximum filtering rates at low resource availability for susceptible and competitor species respectively, and $f_{S 1}$ and $f_{C 1}$ determine how rapidly their foraging rates decline in response to increasing resource availability

$$
\begin{aligned}
& f_{S}(R)=\frac{f_{S 0}}{1+f_{S 1} R}=\frac{0.02}{1+4 R} \\
& f_{C}(R)=\frac{f_{C 0}}{1+f_{C 1} R}=\frac{0.025}{1+4 R}
\end{aligned}
$$

The pathogen basic reproduction number $\left(R_{0}\right)$ is a threshold quantity determining pathogen invasion. We provide it to highlight the effect of the opposing forces of spore removal through foraging $\left(f_{S}\right.$ and $\left.z_{S}\right)$, and spore creation through pathogen transmission $(u)$ and infected host death $(\theta)$. For our system, this can be expressed as

$$
R_{0}=\frac{\theta u f_{S}\left(R_{S}\right) S^{*}}{\mu_{P}+z_{S} f_{S}\left(R_{S}\right) S^{*}}
$$

with the derivation outlined in the Appendix S1.

Parameter definitions, units, and details of the parametrization are provided in Table 1; parameter values were obtained largely from the published literature. To account for uncertainty in some parameter estimates, and to investigate the generality of the simulation results, we performed a sensitivity analysis (Appendix S1). We solved this model numerically for a range of initial competitor densities (0-100 hosts/L). Simulations were initiated with 30 susceptible hosts, no infected hosts, and 10000 pathogen spores. Simulated epidemics were run for $70 \mathrm{~d}$, corresponding to conditions in experimental epidemics. From epidemic simulations, we calculated mean infection prevalence (i.e., average infection prevalence over 70-d time series), and mean susceptible host density as our response variables to changes in competitor density.

\section{Experimental epidemics}

To test our model predictions, we devised a mesocosm experiment where we manipulated competitor density as a means to modify resource availability, and therefore the effects of competition. Experimental populations were formed by dividing five gallon glass aquaria $(16 \prime \times 8 \prime \prime \times 10 \prime)$ in half, separating the two sides of the aquaria with partitions of $210 \mu \mathrm{m}$ Nitex mesh, and filling the tank with $6 \mathrm{~L}$ of media; a combination of $2 \mathrm{~L}$ filtered pondwater $(30 \mu \mathrm{m}$ filter $)$ and $4 \mathrm{~L}$ deionized water. Species were separated by this mesh partition, which allowed for the flow of resources and pathogen spores, but restricted movement of individuals, thereby isolating the effects of resource competition (i.e., exploitative competition) from any direct interaction (i.e., interference competition), and removing any confusion identifying Daphnia neonates to species.

Resource competition was produced by altering the density of $D$. pulicaria and restricting algal resources. Every day, each half of experimental mesocosms was fed $1 \mathrm{~mL}$ of a solution of $200 \mathrm{mg}$ freeze-dried, pulverized Spirulina sp. suspended in $100 \mathrm{~mL}$ deionized water. We fed both partitions of the aquaria the same amount to ensure that resources were well-mixed between halves of each tank, and that the resource concentration throughout the aquaria was approximately $0.67 \mathrm{mg}$ algal dry mass/L. Five mesocosms were formed for each of three initial $D$. pulicaria densities $(0,30$, and 100 individuals/L) for a total of 15 aquaria. Populations of $D$. dentifera were established in each of the 15 experimental aquaria at a density of 30 individuals/L at the start of the experiment. Competitor densities were chosen based on our susceptible host density, where the 
30 individual/L treatment corresponds to both species starting at equal densities, and the 100 competitors/L corresponding to a case where the competitor dominates the community. Both sides of the aquaria were inoculated with 10 Metschnikowia spores/mL $1 \mathrm{~d}$ after populations were established.

Mesocosms were sampled every 3-4 d until infection was no longer observed, which was after $70 \mathrm{~d}$. We assessed infection prevalence and host density by stirring tanks and taking a 1-L water sample from each partition of each aquarium. Infection was assessed by visual inspection using a dissecting microscope (10-40×) under low light and keeping hosts in a minimal amount of water to reduce host mortality. Hosts are translucent, and opaque pathogen clusters are present in host heart or thoracic limbs approximately 1 week after pathogen transmission. Hosts were returned to their respective aquarium. Sampling with replacement is ideal in this experiment, as spores that infected hosts liberate upon death drive subsequent infections in natural systems; removal of infected individuals would artificially reduce epidemic size or duration.

We analyzed the influence of competition on epidemic dynamics and host density. To examine epidemic dynamics in $D$. dentifera in response to competition with D. pulicaria, we calculated two quantities meant to capture aspects of epidemic size and duration: mean infection prevalence and epidemic duration. Mean infection prevalence was quantified as the fraction of $D$. dentifera infected averaged over the total number of sampling points in which the susceptible host population persisted. Epidemic duration was defined as the number of days epidemics had non-zero prevalence. These measures were compared among initial competitor density treatments using Kruskal-Wallis tests. These tests addressed the influence of competitor density on infection dynamics and epidemic duration. Kruskal-Wallis tests were also used to investigate the relationship between the time until $D$. dentifera population extinction and initial competitor density, which addressed the influence of competitor density on susceptible host demography and extinction dynamics. While it is possible that very small populations would not be detected in our 1-L sample, population extinction was noted only when a sample contained no hosts, and a visual inspection of the tank confirmed no living $D$. dentifera hosts.

\section{RESULTS}

\section{Comparison of model and experiments}

Equilibrium analysis of the model (see Appendix S1) demonstrated that in the long term, D. dentifera would be excluded by $D$. pulicaria, and indeed our experimental populations went extinct within $70 \mathrm{~d}$. Our epidemiological model revealed some outcomes that were not observed in our experimental epidemics. For instance, by examining numerous algal resource input values (Figs. 1 and 2), we found that the theoretical hump-shaped relationship between initial competitor density and infection prevalence in the susceptible host species was not strongly influenced by resource availability. Consideration of the pathogen basic reproductive number in the absence of the competitor suggested that the addition of a competitor could enhance or reduce epidemic risk through antagonistic effects of increasing the filtering rate (and the chance of pathogen exposure) while simultaneously reducing the number of susceptible hosts and infectious propagules. Further exploration of the conditions where competition could reduce or enhance epidemic risk is outlined in the sensitivty analysis section of the Appendix S1. Overall, this effort suggested that the hump-shaped relationship between competitor density and infection prevalence observed in both our experimental epidemics and epidemiological model is robust to a range of parameter values. The range of parameters in which competitor density strictly reduces infection prevalence is small, and corresponds to situations in which the competitor digests a much larger proportion of spores than the susceptible host, or when susceptible hosts produce too few infectious spores to result in sustained transmission. Infection prevalence in the susceptible host species increased when competitors were first added to the system until a threshold was reached, and then declined. When resources were less limiting, competitors were able to reduce infection prevalence in the susceptible host more strongly, and mean susceptible host population sizes were larger (Fig. 2).

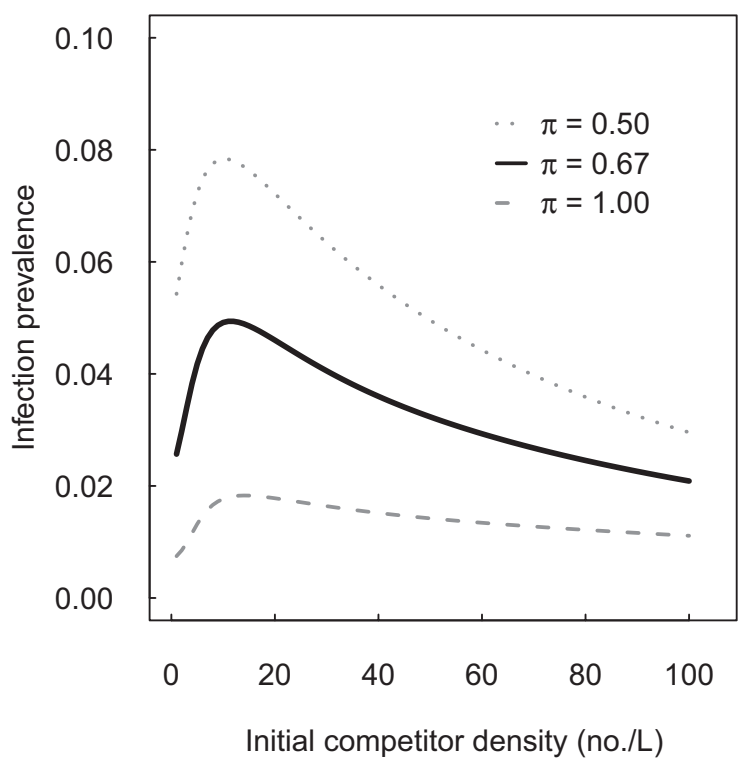

FIG. 1. Non-monotonic relationship between mean infection prevalence and initial competitor density ( $x$-axis) for three potential algal resource input levels $(\pi)$. Enhanced resource input rates reduce infection prevalence by decreasing filtering rates (and hence pathogen exposure). The reduction in infection prevalence at larger initial competitor densities is a result of reduced susceptible host population sizes, and not a positive effect of the competitor removing environmental pathogen. 


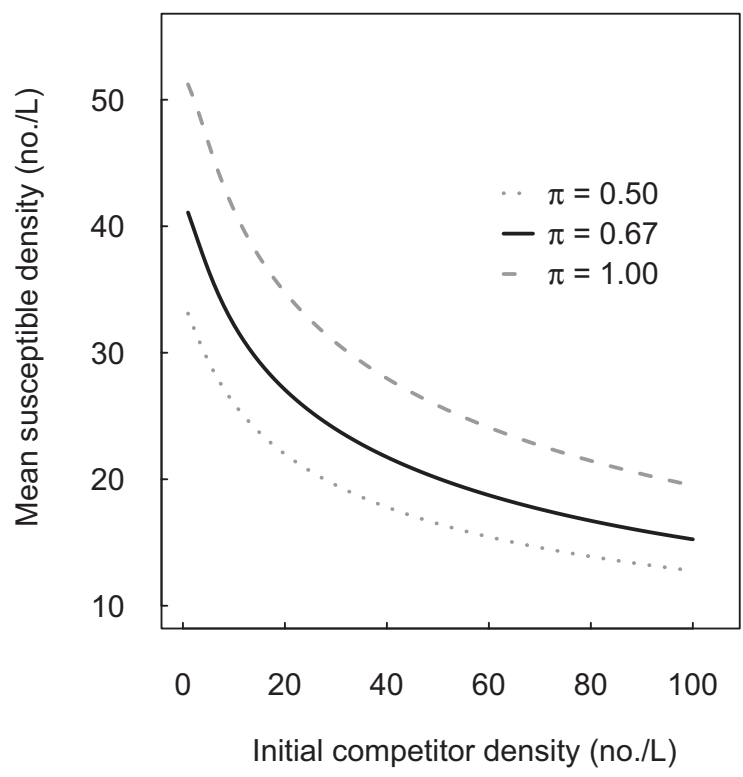

FIG. 2. Mean population size of the susceptible host species $(S+I)$ as a function of initial competitor density for three different algal resource input levels. $\pi=0.5$ and $\pi=1$ are the lower and upper dashed lines, respectively, and the solid black line corresponds to $\pi=0.67$, the rate of algal resource supply in our experimental epidemics.

\section{Competitor density and susceptible host epidemic dynamics}

Experimental epidemics were qualitatively similar to predictions derived from our epidemiological model (Fig. 1), despite independent parameterization of the epidemiological model. The first testable prediction from our theoretical model was that prevalence has a hump-shaped relationship with the initial density of the competitor species. In our experimental epidemics, competitor density had a strong effect on susceptible host species infection dynamics (Figs. 3 and 4). Mean infection prevalence (Kruskal-Wallis test; $\chi^{2}=6.74$, $\mathrm{df}=2, P=0.034)$ and epidemic duration (KruskalWallis test; $\chi^{2}=6.31, \mathrm{df}=2, P=0.043$ ) both increased at intermediate levels of competition (30 Daphnia/L for both species). Further, it is interesting to note that at the early stages of epidemics, after Daphnia populations were exposed to free-living pathogen spores, infection prevalence increased monotonically with competitor density (Fig. 3), suggesting that the competitor presence increased infection prevalence over very short timescales.

\section{Competitor density reduces susceptible host population size}

Our model predicts that mean susceptible host population size should decline with increasing initial competitor density (Fig. 2). In our experiments, epidemics were smaller when $D$. pulicaria densities were at their highest (100 D. pulicaria/L), driven not by the removal of pathogen from the environment, but by the competitive exclusion of the inferior competitor (Fig. 4B). The time until $D$. dentifera extinction was reduced by increasing $D$. pulicaria density, though not significantly (Kruskal-Wallis test; $\chi^{2}=4.92$, df $=2, P=0.085$ ). The resulting termination of epidemics with competitive exclusion is evident when examining the infection time series (Fig. 3). However, experimental epidemics also resulted in susceptible host extinction in the absence of

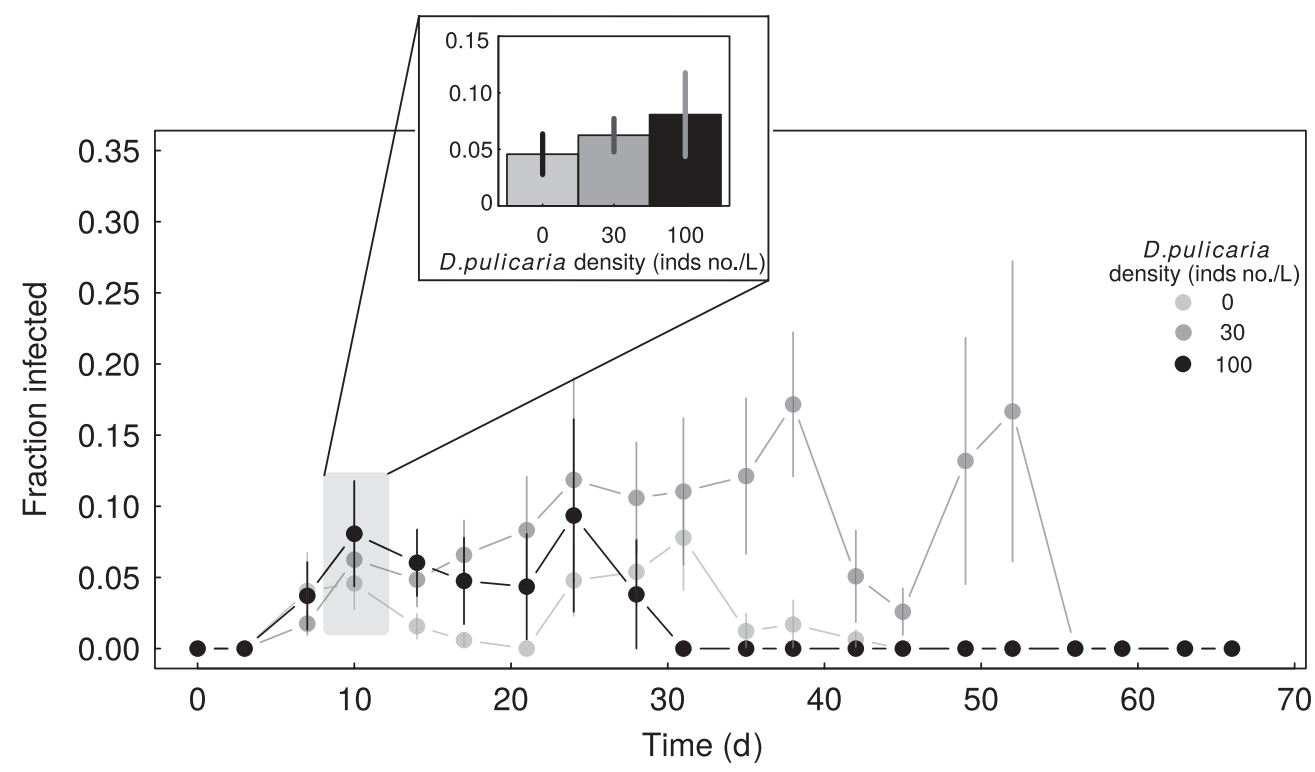

FIG. 3. Infection prevalence (mean and SE) for the epidemic time series. The inset barplot compares the infection prevalence at day 10 , which corresponds to the first wave of infection, as the pathogen typically takes between 7 and $12 \mathrm{~d}$ to be readily identifiable, suggesting that competition intitially increased infection prevalence proportional to Daphnia pulicaria density. 

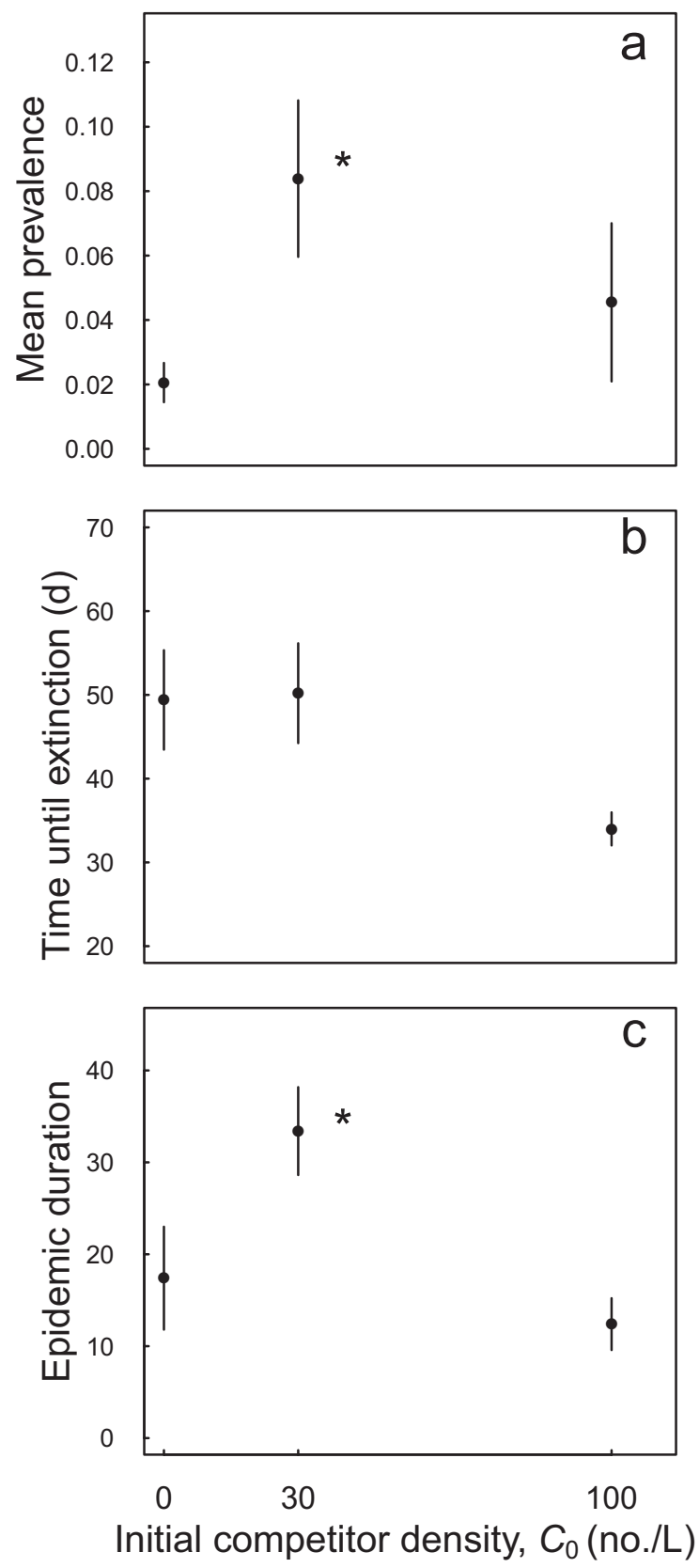

FIG. 4. Experimental epidemics at three competitor densities revealed that intermediate levels of competition significantly increased (A) mean infection prevalence and (C) epidemic duration, and reduced (B) time until extinction of susceptible host populations. Plotted points are means and SE. There was no difference in epidemic measures between no competitor and high $D$. pulicaria competitor density treatments. Asterisks indicate a significant difference as a function of competitor density $(\alpha=0.05)$.

competition, a phenomenon not predicted in our model. This is likely an experimental artifact; a result of detriorating water quality, limited food supply, and enhanced mortality as a result of twice a week sampling.

\section{DisCUSSION}

This study investigates support for the "friendly competition" concept (Hall et al. 2009) over ecologically relevant timescales, using a theoretical model and experiments. We found that when resources are limiting, competition with a superior competitor may be entirely "unfriendly" to susceptible host populations in two different ways: by increasing infection prevalence in susceptible hosts (through increased filtering induced by nutritional stress), and by reducing resources to levels below which susceptible host populations cannot maintain themselves. Prevalence increases through increased pathogen intake occurred at intermediate competitor densities; although prevalence declined at high competitor densities (often interpreted as evidence for friendly competition), the net effect of competition was negative, reducing susceptible host populations toward competitive exclusion. Consistent with classic theory (Gause 1934), our model predicts that eventual competitive exclusion of the inferior competitor is inevitable, barring niche partitioning or other coexistence mechanisms such as refugia. Overall, our findings suggest that the competitive effects of a dominant competitor are usually negative, and that any positive effect of the competitor removing pathogen from the environment may be overwhelmed by the effect of reduced resource availability on inferior competitor feeding behavior and persistence.

Our model and experimental design make several simplifying assumptions that could influence competitor effects on infection dynamics in natural systems. For instance, our model allowed filtering rate, which is crucial to pathogen transmission, to vary only with resource quantity while other factors (e.g., pesticides; FernándezCasalderrey, Ferrando and Andreu-Moliner 1994) may influence foraging ecology and therefore affect transmission independently of resource depletion by competitors. Within hosts, gut residence time may correspond to changes in the probability that a pathogen spore will pierce the gut wall and cause infection. This might explain the findings of previous studies in which both biotic and abiotic stressors decreased filtering rate and also increased pathogen infection success (Day and Kaushik 1987; Fernández-Casalderrey, Ferrando and Andreu-Moliner 1994; Coors and De Meester 2008; Coors et al. 2008; Jansen et al. 2011). Hosts experiencing stress, either through starvation (Pulkkinen and Ebert 2004) or from the presence of secondary compounds from competitors, may experience higher transmission success due to the inability to resist pathogen infection (Lafferty and Holt 2003); in this case, prevalence may continue to increase at higher competitor densities than predicted by our model. Finally, Daphnia feeding selectivity (DeMott 1982; Knisely and Geller 1986), and spatial aggregation of pathogen (given that pathogen spores settle quickly after host mortality) may reduce spore encounter rates and host infection independently of resource and competitor density. 
Previous studies in the Daphnia-microparasite system have suggested that competition with a non-susceptible host should reduce infection prevalence, resulting in so-called "friendly competition" (Hall et al. 2009). Further, Civitello et al. (2013) argued that increasing susceptible host density could inhibit disease spread as a result of pathogen consumption and host foraging interference, suggesting another instance of foraging influencing infection prevalence. Lastly, Cáceres et al. (2014) examined the conditions under which "friendly competition" could result in long term persistence of the inferior competitor, using an epidemiological model nearly identical to ours. We arrived at some conclusions also supported by Cáceres et al. (2014), including the fact that two hosts competing for a limiting resource are unlikely to coexist indefinitely. However, our study also considers the transient dynamics before the susceptible host was excluded. These transient dynamics are ecologically relevant (Hastings 2004), both to zooplankton specifically and to studies of host-parasite interactions more generally (Dobson 2004). Zooplankton populations are unlikely to have equilibrium population densities (McCauley and Murdoch 1987) due to seasonal and stochastic changes in resource availability and environmental conditions, which influence host demographic rates. This means that models examining equilibrium conditions may not correspond to experimental data, making comparisons of models to experiments difficult (Hastings 2004). Our analysis suggests that friendly competition is unlikely to occur over shorter, biologically relevant timescales.

There are many ways that parasites can influence interactions between hosts (Hatcher, Dick and Dunn 2012). Many studies focus on how a parasite can handicap the superior competitor, leading to parasite-mediated coexistence (Freeland 1983; Schall 1992; Schmitz and Nudds 1994; Hatcher, Dick and Dunn 2006). However, these studies typically do not consider how pathogen uptake is influenced by changes to foraging rates due to basal resource availability. Our study suggests that competition-mediated foraging rates could increase prevalence in a pathogen-susceptible, superior resource competitor, reducing its abundance relative to the inferior competitor below that expected when pathogen transmission is assumed to be independent of resource availability. Further theoretical and empirical work in this area is warranted.

Given its importance for transmission potential of zoonoses such as Lyme Disease (Ostfeld and Keesing 2012), there has been much recent interest in the role of host diversity in either diluting or amplifying pathogen transmission (Civitello et al. 2015). Many studies of diversitydisease relationships tend not to incorporate ecological interactions, most notably competition for basal resources. In simple systems where a host and non-host diluter acquire environmental pathogen stages, prevalence is predicted to decline monotonically with non-host density. Our results show that at least initially, intermediate diluter density maximizes epidemic size by increasing host acquisition of the pathogen. The situation in which the pathogen can become a food resource introduces complexity into the study of infectious disease in ecological communities, but is not specific to our study system. Many pathogens are environmentally transmitted, and are subject to incidental predation by hosts, and non-hosts alike (Thieltges et al. 2008; Parker, Elderd and Dwyer 2010). Therefore, our results suggest that evidence for dilution or amplification may be influenced by the time scale of observation, resource availability, as well as the relative abundance or richness of lower-competency hosts.

Much like Strauss et al. (2015), this study attempts to unify two concepts in disease ecology by relating dilution theory to parasite-mediated competition. We highlight the importance of ecological context (resource availability) to competitive interactions between hosts, and how this influences infection dynamics in the susceptible host through a mechanism related to host foraging ecology. Studies of diversity-disease relationships and parasite-mediated competition often do not incorporate the potentially strong effect of environmental regulation, specifically with regard to resource availability. The incorporation of resource-mediated species interactions (direct and indirect) into studies of diversity-disease relationships may yield a more mechanistic view of diversity-disease relationships and other areas of disease ecology.

\section{ACKNOWLEDGMents}

We thank Brett Elderd and an anonymous reviewer for their thoughtful suggestions. Funding for this study was provided by the University of Georgia's Odum School of Ecology.

\section{Literature Cited}

Armstrong, R. A., and R. McGehee. 1976. Coexistence of species competing for shared resources. Theoretical Population Biology 9:317-328.

Auld, S., R. Penczykowski, J. Housley Ochs, D. Grippi, S. Hall, and M. Duffy. 2013. Variation in costs of parasite resistance among natural host populations. Journal of Evolutionary Biology 26:2479-2486.

Cáceres, C., G. Davis, S. Duple, S. Hall, A. Koss, P. Lee, and Z. Rapti. 2014. Complex Daphnia interactions with parasites and competitors. Mathematical Biosciences 258:148-161.

Carius, H. J., T. J. Little, and D. Ebert. 2001. Genetic variation in a host-parasite association: potential for coevolution and frequency-dependent selection. Evolution 55:1136-1145.

Carmona-Catot, G., K. Magellan, and E. García-Berthou. 2013. Temperature-specific competition between invasive mosquitofish and an endangered cyprinodontid fish. PLoS ONE 8:e54734.

Civitello, D. J., S. Pearsall, M. A. Duffy, and S. R. Hall. 2013. Parasite consumption and host interference can inhibit disease spread in dense populations. Ecology Letters 16:626-634.

Civitello, D. J., J. Cohen, H. Fatima, N. T. Halstead, J. Liriano, T. A. McMahon, C. N. Ortega, E. L. Sauer, T. Sehgal, S. Young, et al. 2015. Biodiversity inhibits parasites: broad evidence for the dilution effect. Proceedings of the National Academy of Sciences USA 112:8667-8671.

Coors, A., E. Decaestecker, M. Jansen, and L. De Meester. 2008. Pesticide exposure strongly enhances parasite virulence in an invertebrate host model. Oikos 117:1840-1846. 
Coors, A., and L. De Meester. 2008. Synergistic, antagonistic and additive effects of multiple stressors: predation threat, parasitism and pesticide exposure in Daphnia magna. Journal of Applied Ecology 45:1820-1828.Dallas, T., and J. M. Drake. 2014. Nitrate enrichment alters a Daphnia-microparasite interaction through multiple pathways. Ecology and Evolution $4: 243-250$.

Day, K., and N. Kaushik. 1987. Short-term exposure of zooplankton to the synthetic pyrethroid, fenvalerate, and its effects on rates of filtration and assimilation of the alga, Chlamydomonas reinhardii. Archives of Environmental Contamination and Toxicology 16:423-432.

Decaestecker, E., D. Verreydt, L. De Meester, and S. A. Declerck. 2015. Parasite and nutrient enrichment effects on Daphnia interspecific competition. Ecology 96:1421-1430.

DeMott, W. R. 1982. Feeding selectivities and relative ingestion rates of Daphnia and Bosmina. Limnology and Oceanography 27:518-527.

Dobson, A. 2004. Population dynamics of pathogens with multiple host species. American Naturalist 164:S64-S78.

Duffy, M. A. 2009. Staying alive: the post-consumption fate of parasite spores and its implications for disease dynamics. Limnology and Oceanography 54:770-773.

Duffy, M. A., and S. R. Hall. 2008. Selective predation and rapid evolution can jointly dampen effects of virulent parasites on Daphnia populations. American Naturalist 171:499-510.

Duffy, M. A., C. E. Cáceres, S. R. Hall, A. J. Tessier, and A. R. Ives. 2010. Temporal, spatial, and between-host comparisons of patterns of parasitism in lake zooplankton. Ecology 91:3322-3331.

Engel, K., and R. Tollrian. 2011. Competitive ability, thermal tolerance and invasion success in exotic Daphnia lumholtzi. Journal of Plankton Research 34:92-97.

Fernández-Casalderrey, A., M. Ferrando, and E. Andreu-Moliner. 1994. Effect of sublethal concentrations of pesticides on the feeding behavior of Daphnia magna. Ecotoxicology and Environmental Safety 27:82-89.

Freeland, W. 1983. Parasites and the coexistence of animal host species. American Naturalist 121:223-236.

Gause, G. 1934. The struggle for existence. Williams and Wilkins, Baltimore, Maryland, USA.

Gliwicz, Z. M. 2004. Zooplankton. Pages 461-516 in P. O'Sullivan and C. Reynolds, editors. The lakes handbook, limnology and limnetic ecology. Volume 1. Wiley-Blackwell, Oxford, UK.

Hall, S. R., L. Sivars-Becker, C. Becker, M. A. Duffy, A. J. Tessier, and C. E. Cáceres. 2007. Eating yourself sick: transmission of disease as a function of foraging ecology. Ecology Letters 10:207-218.

Hall, S. R., C. R. Becker, J. L. Simonis, M. A. Duffy, A. J. Tessier, and C. E. Cáceres. 2009. Friendly competition: evidence for a dilution effect among competitors in a planktonic hostparasite system. Ecology 90:791-801.

Hall, S. R., C. R. Becker, M. A. Duffy, and C. E. Cáceres. 2010. Variation in resource acquisition and use among host clones creates key epidemiological trade-offs. American Naturalist 176:557-565.

Hastings, A. 2004. Transients: the key to long-term ecological understanding? Trends in Ecology and Evolution 19:39-45.

Hatcher, M. J., J. T. Dick, and A. M. Dunn. 2006. How parasites affect interactions between competitors and predators. Ecology Letters 9:1253-1271.

Hatcher, M. J., J. T. Dick, and A. M. Dunn. 2012. Diverse effects of parasites in ecosystems: linking interdependent processes. Frontiers in Ecology and the Environment 10:186-194.
Hu, S. S., and A. J. Tessier. 1995. Seasonal succession and the strength of intra- and interspecific competition in a Daphnia assemblage. Ecology 76.7:2278-2294.

Hutchinson, G. E. 1961. The paradox of the plankton. American Naturalist 95:137-145.

Jansen, M., A. Coors, R. Stoks, and L. De Meester. 2011. Evolutionary ecotoxicology of pesticide resistance: a case study in Daphnia. Ecotoxicology 20:543-551.

Keesing, F., R. D. Holt, and R. S. Ostfeld. 2006. Effects of species diversity on disease risk. Ecology Letters 9:485-498.

Kilpatrick, A. M., P. Daszak, M. J. Jones, P. P. Marra, and L. D. Kramer. 2006. Host heterogeneity dominates West Nile virus transmission. Proceedings of the Royal Society B 273:2327-2333.

King, K. C., S. K. Auld, P. J. Wilson, J. James, and T. J. Little. 2013. The bacterial parasite Pasteuria ramosa is not killed if it fails to infect: implications for coevolution. Ecology and Evolution 3:197-203.

Knisely, K., and W. Geller. 1986. Selective feeding of four zooplankton species on natural lake phytoplankton. Oecologia 69:86-94

Lafferty, K. D., and R. D. Holt. 2003. How should environmental stress affect the population dynamics of disease? Ecology Letters 6:654-664.

McCauley, E., and W. W. Murdoch. 1987. Cyclic and stable populations: plankton as paradigm. American Naturalist 129:97-121.

Orlofske, S. A., R. C. Jadin, D. L. Preston, and P. T. Johnson. 2012. Parasite transmission in complex communities: predators and alternative hosts alter pathogenic infections in amphibians. Ecology 93:1247-1253.

Ostfeld, R. S., and F. Keesing. 2012. Effects of host diversity on infectious disease. Annual Review of Ecology, Evolution, and Systematics 43:157.

Park, T. 1948. Interspecies competition in populations of Trilobium confusum Duval and Trilobium castaneum Herbst. Ecological Monographs 18:265-307.

Parker, B. J., B. D. Elderd, and G. Dwyer. 2010. Host behaviour and exposure risk in an insect-pathogen interaction. Journal of Animal Ecology 79:863-870.

Pickett, S. T. 1980. Non-equilibrium coexistence of plants. Bulletin of the Torrey Botanical Club 107:238-248.

Porter, K. G., J. Gerritsen, and J. D. Orcutt Jr 1982. The effect of food concentration on swimming patterns feeding, behavior ingestion, assimilation, and respiration, by Daphnia. Limnology and Oceanography 27:935-949.

Preston, D., and P. Johnson. 2010. Ecological consequences of parasitism. Nature Education Knowledge 1:39.

Price, P. W., M. Westoby, B. Rice, P. R. Atsatt, R. S. Fritz, J. N. Thompson, and K. Mobley. 1986. Parasite mediation in ecological interactions. Annual Review of Ecology and Systematics 17:487-505

Price, P. W., M. Westoby, and B. Rice. 1988. Parasite-mediated competition: some predictions and tests. American Naturalist 131:544-555.

Pulkkinen, K., and D. Ebert. 2004. Host starvation decreases parasite load and mean host size in experimental populations. Ecology 85:823-833.

Riebesell, J. F. 1974. Paradox of enrichment in competitive systems. Ecology 55:183-187.

Salkeld, D. J., K. A. Padgett, and J. H. Jones. 2013. A meta-analysis suggesting that the relationship between biodiversity and risk of zoonotic pathogen transmission is idiosyncratic. Ecology Letters 16:679-686.

Sarnelle, O., and A. E. Wilson. 2008. Type III functional response in Daphnia. Ecology 89:1723-1732. 
Schall, J. J. 1992. Parasite-mediated competition in Anolis lizards. Oecologia 92:58-64.

Scheffer, M., S. Rinaldi, J. Huisman, and F. J. Weissing. 2003. Why plankton communities have no equilibrium: solutions to the paradox. Hydrobiologia 491:9-18.

Schmitz, O. J., and T. D. Nudds. 1994. Parasite-mediated competition in deer and moose: how strong is the effect of meningeal worm on moose? Ecological Applications 4:91-103.

Searle, C., J. Ochs, C. Cáceres, S. Chiang, N. Gerardo, S. Hall, and M. Duffy. 2015. Plasticity, not genetic variation, drives infection success of a fungal parasite. Parasitology 142:1-10.

Smith, F. E. 1963. Population dynamics in Daphnia magna and a new model for population growth. Ecology 44:651-663.

Stich, H. B., and G. Maier. 2007. Distribution and abundance of Daphnia pulicaria, a large Daphnia of the "pulex group", in Lake Constance (Lower Lake). Limnologica Ecology and Management of Inland Waters 37:303-310.

Strauss, A. T., D. J. Civitello, C. E. Cáceres, and S. R. Hall. 2015. Success, failure and ambiguity of the dilution effect among competitors. Ecology Letters 18:916-926.
Tessier, A. J., and P. Woodruff. 2002. Cryptic trophic cascade along a gradient of lake size. Ecology 83:1263-1270.

Thieltges, D., M. Bordalo, A. Caballero Hernández, K. Prinz, and K. Jensen. 2008. Ambient fauna impairs parasite transmission in a marine parasite-host system. Parasitology 135:1111-1116.

Thomas, F., J.-F. Guégan, Y. Michalakis, and F. Renaud. 2000. Parasites and host life-history traits: implications for community ecology and species co-existence. International Journal for Parasitology 30:669-674.

Wang, H., K. Dunning, J. J. Elser, and Y. Kuang. 2009. Daphnia species invasion, competitive exclusion, and chaotic coexistence. DCDS-B 12:481-493.

Wedin, D., and D. Tilman. 1993. Competition among grasses along a nitrogen gradient: initial conditions and mechanisms of competition. Ecological Monographs 63: 199-229.

Wollrab, S., A. M. de Roos, and S. Diehl. 2013. Ontogenetic diet shifts promote predator-mediated coexistence. Ecology 94:2886-2897.

\section{SUPPORTING INFORMATION}

Additional supporting information may be found in the online version of this article at http://onlinelibrary.wiley.com/ doi/10.1890/15-0305.1/suppinfo 